\title{
Clinical characterization and risk factors associated with cytokine release syndrome induced by COVID-19 and chimeric antigen receptor T-cell therapy
}

\author{
Ruimin Hong ${ }^{1,2,3,4} \cdot$ Houli Zhao ${ }^{1,2,3,4} \cdot$ Yiyun Wang ${ }^{1,2,3,4} \cdot$ Yu Chen ${ }^{5}$ Hongliu Cai ${ }^{6}$ Yongxian Hu (D) ${ }^{1,2,3,4}$. \\ Guoqing Wei ${ }^{1,2,3,4} \cdot$ He Huang (iD ${ }^{1,2,3,4}$
}

Received: 25 June 2020 / Revised: 21 August 2020 / Accepted: 7 September 2020 / Published online: 17 September 2020

(c) The Author(s), under exclusive licence to Springer Nature Limited 2020

\begin{abstract}
An excessive immune response during coronavirus disease (COVID-19) can induce cytokine release syndrome (CRS), which is associated with life-threatening complications and disease progression. This retrospective study evaluated the clinical characteristics of severe CRS (sCRS, grade 3-4) induced by severe COVID-19 (40 patients) or chimeric antigen receptor T-cell (CAR-T) therapy as a comparator (41 patients). Grade 4 CRS was significantly more common in the COVID19 group $(15 / 40(35.7 \%)$ vs. $5 / 41(12.2 \%), P=0.008)$. The CAR-T group had more dramatic increase in cytokines, including IL-2, IL-6, IL-10, and IFN- $\gamma$. Interestingly, COVID-19 group had significantly higher levels for TNF- $\alpha$ (31.1 pg/ $\mathrm{ml}(16.1-70.0)$ vs. $3.3(1.8-9.6), P<0.001)$ and $\lg$ viral loads were correlated with $\lg$ IL-6 $\left(R^{2}=0.101 ; P<0.001\right)$ and lg IL$10\left(R^{2}=0.105 ; P<0.001\right)$. The independent risk factor for COVID-19-related sCRS was hypertension history (OR: 4.876, 95\% CI: 2.038-11.668; $P<0.001)$. Our study demonstrated that there were similar processes but different intensity of inflammatory responses of sCRS in COVID-19 and CAR-T group. The diagnose and management of severe COVID-19related sCRS can learn lessons from treatment of sCRS induced by CAR-T therapy.
\end{abstract}

\section{Introduction}

Cytokine release syndrome (CRS) is a potentially lifethreatening syndrome that can be caused by viral infection, auto-immune disease, administration of antibody-based

These authors contributed equally: Ruimin Hong, Houli Zhao, Yiyun Wang

Supplementary information The online version of this article (https:// doi.org/10.1038/s41409-020-01060-5) contains supplementary material, which is available to authorized users.

Yongxian $\mathrm{Hu}$

huyongxian2000@aliyun.com

$\triangle$ Guoqing Wei

Weiguoqing2000@sina.com

$\triangle$ He Huang

huanghe@zju.edu.cn

1 Bone Marrow Transplantation Center, the First Affiliated Hospital, School of Medicine, Zhejiang University, Hangzhou, China

2 Institute of Hematology, Zhejiang University, Hangzhou, China therapy, and immunotherapy. This syndrome is characterized by dramatically increased levels of cytokines and immune system dysregulation [1-3]. There is normally a balance between anti-inflammatory and pro-inflammatory cytokines, however, an excessively activated immune response can lead to substantially increased secretion of pro-inflammatory cytokines from lymphocytes ( $\mathrm{T}$ cells, B cells, and natural killer cells) and myeloid cells (monocytes, macrophages, and dendritic cells) $[4,5]$. The clinical presentation of CRS varies according to severity, with mild disease being linked to slight fever, fatigue, anorexia, nausea, vomiting, and headache, whereas severe disease is

3 Zhejiang Province Engineering Laboratory for Stem Cell and Immunity Therapy, Hangzhou, China

4 Zhejiang Laboratory for Systems \& Precision Medicine, Zhejiang University Medical Center, Hangzhou, China

5 Department of Clinical Laboratory, The First Affiliated Hospital, School of Medicine, Zhejiang University, Hangzhou, China

6 Department of Surgical Intensive Care Unit (SICU), The First Affiliated Hospital, School of Medicine, Zhejiang University, Hangzhou, China 
linked to hypotension, shock, disseminated intravascular coagulation, and even multiple organ dysfunction $[6,7]$.

Increasing use of chimeric antigen receptor T-cell (CART) therapy for hematological malignancies [8-11] has led to a more comprehensive understanding of the mechanisms underlying CAR-T-related CRS. The pathophysiology of CRS in this setting can be divided into two primary steps after binding of CAR-T receptor and its specific antigen $[5,6]$. First, activated CAR-Ts and lytic tumor cells produce tremendous amounts of IFN- $\gamma$ and TNF- $\alpha$, which initiates the CRS. Second, other immune cells are activated by IFN$\gamma$, especially macrophages and monocytes, which also release massive amounts of other cytokines (including IL-1, IL-6, and IL-10) [12, 13]. In addition, endothelial activation may contribute to CRS, as severe CRS (sCRS, grade 3-4) involves increased serum concentrations of von Willebrand factor and angiopoietin-2 [13]. These pathways contribute to the systemic effects in patients with CAR-T-related CRS.

Coronavirus disease (COVID-19) is caused by severe acute respiratory syndrome coronavirus 2 (SARS-COV-2), which has a fatality rate of $\sim 3.7 \%$ in China based on official national statistics [14]. This emerging infectious disease has prompted research regarding its epidemiology and pathophysiological mechanisms, as well as diagnosis and treatment strategies [14-16]. Giuseppe et al. have suggested that dendritic cells and macrophages release cytokines (including IL-12, IL-15, and IL-18) after viral antigen recognition. Furthermore, activated natural killer cells, recruited group 1 innate lymphoid cells, and differentiated type 1 helper cells may enhance the production of cytokines (including IL-1, IL-2, IFN- $\gamma$, and TNF- $\alpha$ ), which can induce injury of respiratory epithelial and endothelial cells and lead to a variety of respiratory symptoms $[17,18]$. Several studies have indicated that critical COVID-19 cases with CRS may rapidly develop life-threatening complications. [19, 20]. Although the pathophysiology of COVID-19-associated CRS remains poorly understood, and appropriate therapies are still being explored, it is possible that COVID-19induced CRS and CAR-T therapy-induced CRS might have similar pathophysiological process. Therefore, we evaluated the clinical and laboratory characteristics of patients with sCRS that was induced by severe COVID-19 or CAR-T therapy, which may help provide practical information to guide the management of COVID-19-related CRS.

\section{Methods}

\section{Patients}

We retrospectively evaluated patients with sCRS that was induced by severe COVID-19 (the COVID-19 group) between January 2020 and March 2020 at The First
Affiliated Hospital, School of Medicine, Zhejiang University. During this period, 105 patients at our center had confirmed SARS-COV-2 infection based on viral nucleic acid detection, which was identified in accordance with the Guidelines and Management of COVID-19 of the National Health Commission of China [21]. The CAR-T group was defined as patients who received CAR-T therapy for refractory/relapsed hematological malignancies between July 2015 and April 2020 at our Bone Marrow Transplantation Center (The First Affiliated Hospital, School of Medicine, Zhejiang University). Cases of CRS were identified based on version 5.0 of the National Cancer Institute Common Terminology Criteria for Adverse Events, which define CRS as a disorder that involves fever, tachypnea, headache, tachycardia, hypotension, rash, and/or hypoxia caused by the release of cytokines $[3,6]$. The present study only included patients with sCRS, which required the patients to fulfill one of the following criteria: (1) fever of $>38^{\circ} \mathrm{C}$, (2) inspired oxygen concentration of $\geq 40 \%$, or (3) hypotension requiring one or more vasopresors [22]. Based on these criteria, the COVID-19 group included 40 patients with sCRS and the CAR-T group included 41 patients with sCRS, and 40 patients in COVID-19 group were severe or critically ill patients. The study was retrospectively registered in the Chinese clinical trial registry (http://www.chictr. org.cn/showproj.aspx ?proj=49177) $($ ChiCTR2000029625) on 07 February 2020 and (http://www.chictr.org.cn/show proj.aspx?proj=11714) (ChiCTR-OCC-15007008) on 04 August 2015.

\section{Laboratory measurements and data collection}

The patients' electronic medical records were searched to collect data regarding their epidemiological, clinical, laboratory, radiographic, and treatment characteristics. The clinical laboratory variables tested after a confirmed diagnosis of COVID-19 during hospitalization included complete blood count, serum biochemical test results (including liver function, renal function, triglycerides, and creatinine), coagulation profile, procalcitonin, ferritin, viral load, and immunological test results (including serum cytokines and peripheral immune cell subsets).

\section{Study endpoints and statistical analysis}

The groups with sCRS induced by severe COVID-19 or CAR-T therapy were compared to identify similarities and differences in their characteristics. The primary endpoints were remission of sCRS and the primary disease as well as mortality. The secondary endpoint was the development of complications (including respiratory failure, cardiac dysfunction, hepatic dysfunction, renal dysfunction, secondary infection, etc). Numeric variables were presented as median 
(interquartile range, IQR) and compared using the Mann-Whitney $U$ test. Categorical variables were presented as number (\%) and compared using Fisher's exact test or the chi-squared test. A Logistic regression model was used to obtain the odds ratio (OR) estimates and corresponding 95\% confidence intervals (CI) to evaluate the risk factors associated with sCRS and clinical complications. Pearson's correlation analysis was used to analyze correlations between the $\mathrm{lg}$ viral load/tumor burden and $\mathrm{lg}$ cytokine levels. Cumulative incidences of sCRS was estimated using the reverse Kaplan-Meier method. Differences were considered statistically significant at two-sided $P$ values of $<0.05$. All analyses were performed using IBM SPSS Statistics (version 24; IBM Corp., Armonk, NY).

\section{Results}

\section{Patient characteristics}

This study included 81 patients with grade 3-4 CRS, who were categorized into a COVID-19 group (40 patients) and a CAR-T group (41 patients). The patients' characteristics are shown in Table 1 . Grade $3 \mathrm{CRS}$ was more common than grade 4 CRS in the COVID-19 group (62.5\% [25/40] vs. $37.5 \%$ [15/40]) and in the CAR-T group (87.8\% [36/41] vs. $12.2 \%$ [5/41]). The incidence of grade 4 CRS was significantly higher in COVID-19 group than in CAR-T group (37.50\% [15/40] vs. $12.2 \%$ [5/41]; $P=0.008)$. The median age was older in COVID-19 group than in CAR-T group (62 years [IQR: 51-71 years] vs. 51 years [IQR: 32.5-62.5 years]; $P<0.001)$. However, there was no significant difference in the median duration (6 days [IQR: 3-31.3 days] vs. 7 days [IQR: $6-10$ days], $P=0.321)$ as well as the cumulative incidence ( $P=0.209$, Supplement Fig. 1) of sCRS between COVID-19 and CAR-T groups.

\section{Clinical symptoms}

The clinical symptoms generally involved respiratory system in COVID-19 group and systemic inflammation in the CAR-T group (Fig. 1 and Table 1). COVID-19 group was more likely to have cough $(95 \%$ [38/40] vs. 53.7\% [22/41], $P<0.001)$, expectoration (70\% [28/40] vs. 36.6\% [15/41], $P=0.003)$, breathing difficulty (95\% [38/40] vs. $48.8 \%$ [20/41], $P<0.001$ ), and hypoxemia (95\% [38/40] vs. $56.1 \%$ [23/41], $P<0.001)$ than CAR-T group. However, the CAR$\mathrm{T}$ group was more likely to have hypotension $(87.8 \%$ [36/ $41]$ vs. $7.5 \%$ [3/40], $P<0.001)$. As fever is an initial sign of CRS, we compared maximum daily temperatures between the two groups. The CAR-T group had a significantly higher median maximum temperature $\left(40.0^{\circ} \mathrm{C}\right.$ [IQR: $39.8-$ $40.2^{\circ} \mathrm{C}$ ] vs. $38.5^{\circ} \mathrm{C}$ [IQR: $\left.\left.37.9-39.0^{\circ} \mathrm{C}\right], P<0.001\right)$ and a
Table 1 Characteristics and clinical symptoms of patients with severe CRS.

\begin{tabular}{|c|c|c|c|}
\hline & $\begin{array}{l}\text { COVID-19 } \\
(N=40)\end{array}$ & $\begin{array}{l}\text { CAR-T } \\
(N=41)\end{array}$ & $P$ value \\
\hline \multicolumn{4}{|l|}{ Characteristics } \\
\hline Age, years & $62(51,71)$ & $51(32.5,62.5)$ & $<0.001$ \\
\hline Gender, $n(\%)$ & & & 0.049 \\
\hline Male & $29(72.5 \%)$ & $21(51.2 \%)$ & \\
\hline Female & $11(27.5 \%)$ & $20(48.8 \%)$ & \\
\hline Grade of CRS, $n(\%)$ & & & 0.008 \\
\hline Grade 3 & $25(62.5 \%)$ & $36(87.8 \%)$ & \\
\hline Grade 4 & $15(37.5 \%)$ & $5(12.2 \%)$ & \\
\hline Duration of CRS, days & $6.0(3.0,31.3)$ & $7.0(6.0,10.0)$ & 0.321 \\
\hline \multicolumn{4}{|l|}{ Clinical symptoms } \\
\hline Max temperature, ${ }^{\circ} \mathrm{C}$ & $38.5(37.9,39.0)$ & $40.0(39.8,40.2)$ & $<0.001$ \\
\hline Duration of fever, days & $8(6,11.3)$ & $10(7,23)$ & 0.014 \\
\hline Cough, $n(\%)$ & $38(95.0 \%)$ & $22(53.7 \%)$ & $<0.001$ \\
\hline Expectoration, $n(\%)$ & $28(70.0 \%)$ & $15(36.6 \%)$ & 0.003 \\
\hline Fatigue, $n(\%)$ & $15(37.5 \%)$ & $22(53.7 \%)$ & 0.144 \\
\hline Headache, $n(\%)$ & $3(7.5 \%)$ & $6(14.6 \%)$ & 0.307 \\
\hline $\begin{array}{l}\text { Blood in phlegm, } \\
n(\%)\end{array}$ & $6(15.0 \%)$ & $3(7.3 \%)$ & 0.271 \\
\hline Diarrhea, $n(\%)$ & $10(25.0 \%)$ & $12(29.3 \%)$ & 0.666 \\
\hline $\begin{array}{l}\text { Breathing difficulties, } \\
n(\%)\end{array}$ & $38(95.0 \%)$ & $20(48.8 \%)$ & $<0.001$ \\
\hline Hypotension, $n(\%)$ & $3(7.5 \%)$ & $36(87.8 \%)$ & $<0.001$ \\
\hline Hypoxemia, $n(\%)$ & $38(95.0 \%)$ & $23(56.1 \%)$ & $<0.001$ \\
\hline
\end{tabular}

Data were described as $\mathrm{n}(\%)$, or median (IQR). $P$ value were tested by Chi-Square test or Mann-Whitney $U$ test. CRS cytokine release syndrome.

longer median duration of fever (10.0 days [IQR: 7.0-23.0 days] vs. 8.0 days [IQR: 6.0-11.3 days], $P=$ $0.014)$.

\section{Laboratory findings}

Figure 2 and Table 2 shows the laboratory parameters that were evaluated for during the course of CRS, including Creactive protein (CRP), cytokine profiles (IL-2, IL-6, IL-10, TNF- $\alpha$, and IFN- $\gamma$ ), D-dimer, triglycerides, procalcitonin, and ferritin. The results revealed that the CAR-T group had significantly higher median maximum values for IL-2, IL-6, IL-10, IFN- $\gamma$, D-dimer, CRP, procalcitonin, and ferritin (Table 2). In addition, TNF- $\alpha$ was the only cytokine with a significantly higher concentration in COVID-19 group than in CAR-T group (31.1 pg/mL [IQR: $16.1-70.0 \mathrm{pg} / \mathrm{mL}] \mathrm{vs}$. $3.3 \mathrm{pg} / \mathrm{mL}$ [IQR: $1.8-9.6 \mathrm{pg} / \mathrm{mL}] ; P<0.001)$, which may provide a meaningful insight into the treatment of sCRS related to COVID-19. Besides, the intervals between maximum cytokines and COVID-19 diagnosis/CAR-T-cell 
Fig. 1 Clinical symptoms in patients with grade 3-4 CRS in COVID-19 group $(n=40)$ and CAR-T group $(n=41)$.

Data were described as $n(\%)$ and median (IQR). Chi-square test and Mann-Whitney $U$ test were used for statistical analysis. Y: yes (had related symptom); $\mathrm{N}$ : no (had no related symptom).
Fig. 2 Peak serum level of cytokine, procalcitonin (PCT), C-reactive protein (CRP), Ddimer (DDI), and ferritin in patients with grade 3-4 CRS in COVID-19 group $(n=40)$ and CAR-T group $(n=41)$. Data were described as median (IQR). The Mann-Whitney $U$ test was used for statistical analysis.

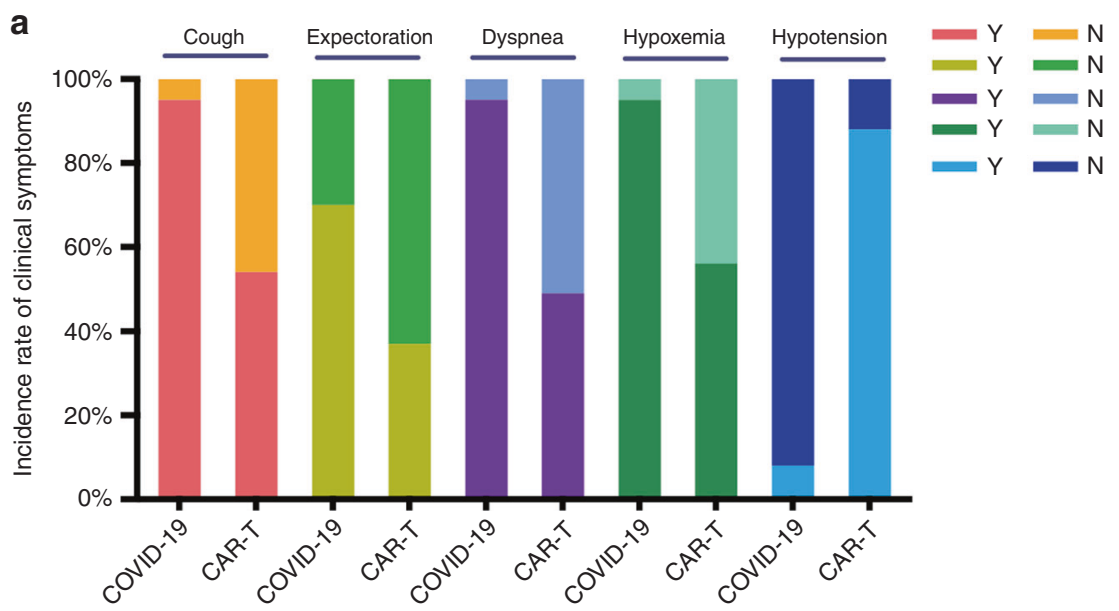

b
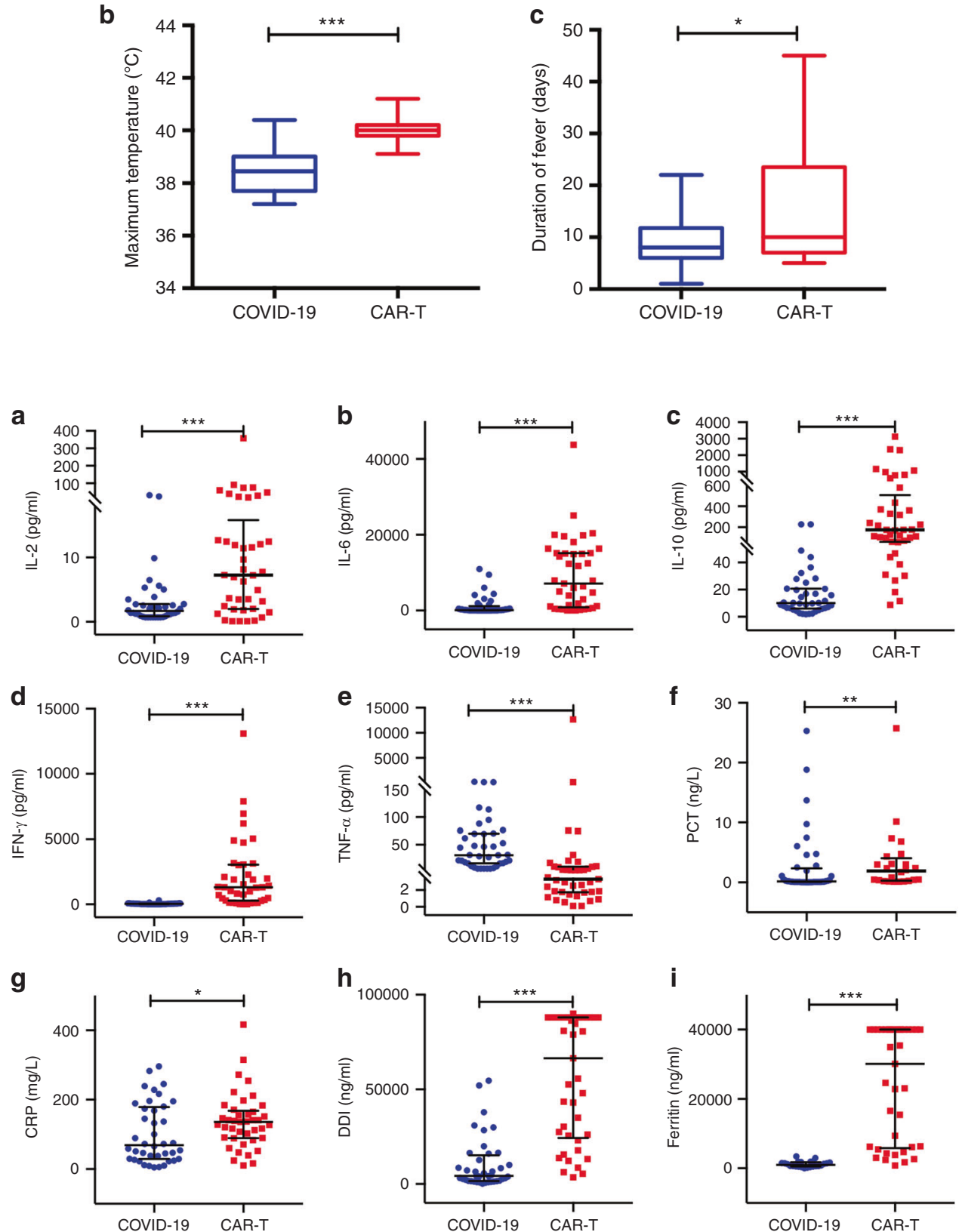
Table 2 Laboratory findings of severe CRS in COVID-19 group and CAR-T group.

$$
\text { COVID-19 }(N=40)
$$

CAR-T $(N=41)$

$P$ value

Laboratory findings

Max CRP, mg/L

$69.0(29.8,175.5)$

Max IL-2, pg/ml

$1.7(0.9,2.7)$

Max IL-6, pg/ml

$110.3(41.7,728.1)$

Max IL-10, pg/ml

$10.1(6.3,20.6)$

Max TNF- $\alpha, p g / m l$

$31.1(16.1,70.0)$

Max IFN- $\gamma, \mathrm{pg} / \mathrm{ml}$

$35.0(16.9,60.8)$

Max D-dimer, ng/ml

Max triglyceride, $\mathrm{mmol} / \mathrm{L}$

$4295.5(1669.5,13522.0)$

$2.6(1.5,3.5)$

$0.2(0.1,2.1)$

Max PCT, ng/L

Max ferritin, ng/ml

$1022.8(538.7,1685.8)$

$$
\begin{aligned}
136.2 & (90.0-165.0) \\
7.3 & (2.0,12.7) \\
7120.6 & (1066.8,15136.4) \\
174.5 & (61.7,434.6) \\
3.3 & (1.8,9.6) \\
1308.5 & (296.6,3018.2) \\
66383 & (25545,88000) \\
2.7 & (2.4,3.8) \\
1.9 & (0.4,3.4) \\
30093.4 & (6147,40000)
\end{aligned}
$$

Intervals between peak cytokines and COVID-19 diagnosis/CAR-T-cell infusion, days

$\begin{array}{lc}\text { IL-2 } & 5.5(1,16) \\ \text { IL-6 } & 6(1,21.75) \\ \text { IL-10 } & 6.5(1,22.5) \\ \text { TNF- } \alpha & 10(1,22) \\ \text { IFN- } \gamma & 9(1,23)\end{array}$

$5(4,8.75)$

$9(5,12.5)$

$10(8,13)$

0.163

$10(7.25,14)$

0.523

Data were described as median (IQR). $P$ value were tested by Mann-Whitney $U$ test. $C R P$ C-reactive protein, $P C T$ procalcitonin. a

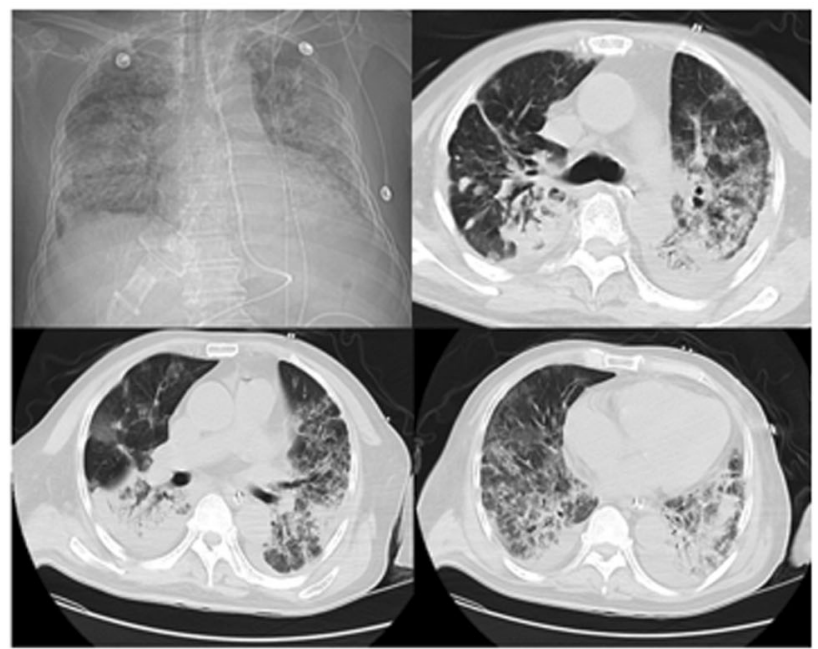

Fig. 3 Imaging features of the lungs in the COVID-19 and CAR-T groups. a Transverse chest CT images from a 74-year-old man with grade 4 CRS in COVID-19 group showing bilateral diffuse groundglass opacity, patchy and streak hyperdensities on day 29 after sCRS

infusion were shown in Table 2, and there were no significant differences between the two groups.

\section{Imaging features of the lungs in the COVID-19 and CAR-T groups}

The imaging features of the lungs in COVID-19 group varied during disease stage. Early-stage disease tended to exhibit multiple patchy ground-glass opacities at the lower b

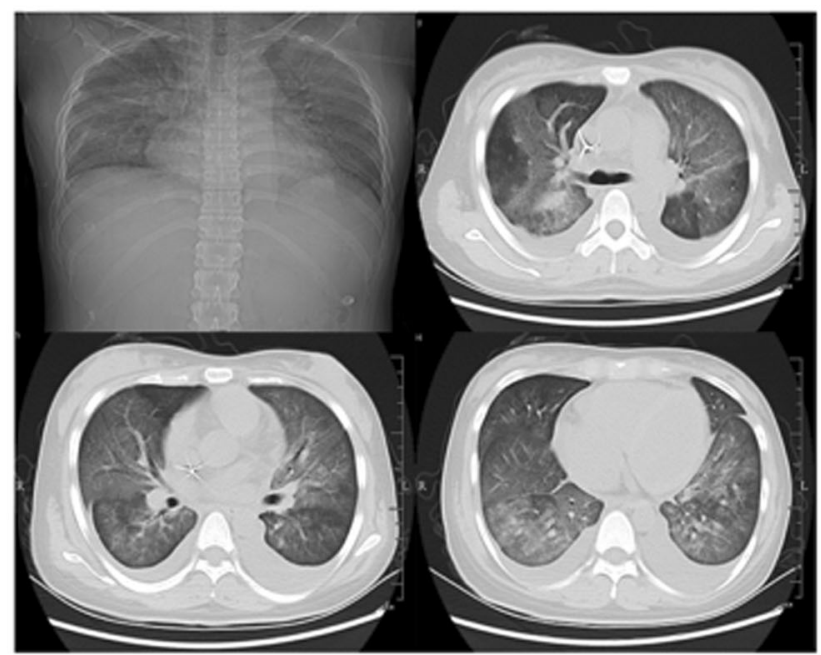

onset. b Transverse chest CT images from a 23-year-old woman with grade 4 CRS in CAR-T group showing bilateral patchy hyperdensities, with uneven internal density and pleural effusion on day 8 after sCRS onset.

lobe and the lateral and sub-pleural regions. Isolated local lesions were less common, although they could progress to bilateral infiltrates within a short time. Mid-to-late-stage disease tended to exhibit coexistence of consolidation affecting the lower lung zone, ground-glass opacities, reticular changes, and focal interstitial fibrosis, although pleural effusion was uncommon (Fig. 3a). The typical imaging feature of the lungs in CAR-T group was diffuse pulmonary infiltration. The radiographic changes in the CAR-T group 
Table 3 Multivariate analyses for risk factors associated with severe CRS and clinical complications.

\begin{tabular}{|c|c|c|c|}
\hline \multicolumn{2}{|l|}{ Factors } & Odds ratio $(95 \% \mathrm{CI})$ & $P$ value \\
\hline \multicolumn{4}{|c|}{ COVID-19-related sCRS } \\
\hline \multicolumn{2}{|l|}{$\begin{array}{l}\text { History of } \\
\text { hypertension }\end{array}$} & $4.876(2.038-11.668)$ & $<0.001$ \\
\hline \multicolumn{4}{|c|}{$C A R$-T-related sCRS in ALL patients } \\
\hline \multicolumn{2}{|l|}{$\begin{array}{l}\text { Blast cells in the } \\
\text { bone marrow }\end{array}$} & $1.025(1.003-1.047)$ & 0.029 \\
\hline \multicolumn{4}{|c|}{$C A R-T$-related $s C R S$ in MM patients } \\
\hline \multicolumn{2}{|l|}{$\beta 2$-microglobulin } & $1.252(1.004-1.560)$ & 0.046 \\
\hline \multicolumn{4}{|c|}{ Clinical complications } \\
\hline \multicolumn{4}{|c|}{ Invasive ventilation } \\
\hline Max triglyceride & & $0.264(0.078-0.895)$ & 0.033 \\
\hline \multicolumn{4}{|l|}{ Respiratory failure } \\
\hline Group & $\begin{array}{l}\text { COVID-19 } \\
\text { vs. CAR-T }\end{array}$ & $\begin{array}{l}175655.306 \\
(121.630-253678007.724)\end{array}$ & 0.001 \\
\hline Max $\lg$ IL-10 & & $88.386(3.309-2360.779)$ & 0.007 \\
\hline \multicolumn{4}{|l|}{ Renal dysfunction } \\
\hline Grade of CRS & $\begin{array}{l}\text { Grade } 4 \text { vs. } \\
\text { grade } 3\end{array}$ & $6.603(1.500-29.078)$ & 0.013 \\
\hline Max CRP & & $1.012(1.004-1.021)$ & 0.003 \\
\hline \multicolumn{4}{|c|}{ Hepatic dysfunction } \\
\hline Max lg ferritin & & $4.181(1.627-10.741)$ & 0.003 \\
\hline \multicolumn{4}{|c|}{ Secondary infection } \\
\hline Max lg D-dimer & & $9.823(1.690-57.113)$ & 0.011 \\
\hline \multicolumn{4}{|l|}{ Blood transfusion } \\
\hline Max lg IL-6 & & $9.948(1.927-51.359)$ & 0.006 \\
\hline
\end{tabular}

Data were described as odds ratio $(95 \% \mathrm{CI}) . P$ value were tested by logistics regression model test. $A L L$ acute lymphoblastic leukemia, $M M$ multiple myeloma, $C R P$ C-reactive protein, $P C T$ procalcitonin.

mainly involved patchy hyperdensities with heterogeneous density, which was occasionally accompanied by pleural effusion and concomitant clinical symptoms (e.g., hyperpyrexia, hypoxemia, and hypotension). Treatment of CRS and lung lesions generally involved comprehensive therapy that consisted of tocilizumab, corticosteroids, and antiinfective agents (Fig. 3b).

\section{Risk factors associated with sCRS}

Table 3 and Supplement Table 1 show the risk factors that were associated with sCRS in COVID-19 and CAR-T groups. The independent risk factors for COVID-19induced sCRS was the history of hypertension (OR: 4.876, 95\% CI: $2.038-11.668 ; P<0.001)$. The risk of COVID-19-induced sCRS was not significantly related to sex, age, history of diabetes, history of cardiovascular disease, history of respiratory disease, smoking status.

The independent risk factors for sCRS in CAR-T group were high levels of bone marrow blast cells among patients with acute lymphoblastic leukemia (ALL) (OR: 1.025, 95\% CI: $1.003-1.047 ; \quad P=0.029)$ and increased $\beta 2$ microglobulin concentrations among patients with multiple myeloma (MM) (OR: 1.252, 95\% CI: 1.004-1.560; $P=$ 0.046). The risk of CAR-T-induced sCRS was not significantly related to sex, age, numbers of prior chemotherapies, prior stem cell transplantation, disease stage, lactate dehydrogenase (LDH) before the CAR-T infusion, or the CAR-T infusion dose in ALL, MM and non-Hodgkin lymphoma (NHL) patients.

\section{Correlations between viral load/tumor burden and cytokine levels}

The correlations between viral load/tumor burden and various cytokine levels were shown in Fig. 4. Results revealed that the $\lg$ viral load was correlated with the $\lg$ serum concentrations of IL-6 $\left(R^{2}=0.101 ; P<0.001\right)$, IL-10 $\left(R^{2}=\right.$ $0.105 ; P<0.001)$, and TNF- $\alpha\left(R^{2}=0.04 ; P=0.005\right)$, but not correlated with the $\mathrm{lg}$ serum concentrations of IL-2 $\left(R^{2}=0.004 ; P=0.359\right)$ or IFN- $\gamma\left(R^{2}<0.001 ; P=0.783\right)$.

In CAR-T group, LDH was a common indicator related to tumor burden among patients with ALL, NHL, and MM. The $\lg \mathrm{LDH}$ concentration was correlated with the $\lg$ serum concentration of IL-6 $\left(R^{2}=0.161 ; P=0.01\right)$ but not with the lg serum concentrations of IL-2 $\left(R^{2}=0.01 ; P=0.548\right)$, IL-10 $\left(R^{2}=0.003 ; P=0.726\right), \quad$ IFN- $\gamma\left(R^{2}=0.001 ; P=\right.$ $0.844)$ or TNF- $\alpha\left(R^{2}=0.003 ; P=0.742\right)$.

\section{Complications and associated risk factors during sCRS}

Table 4 shows the incidences of various complications, including hypoalbuminemia, hepatic dysfunction, renal dysfunction, cardiac dysfunction, respiratory failure, bacterial infection, fungal infection, other viral infection, need for blood transfusion, and gastrointestinal hemorrhage. The COVID-19 group had higher rates of respiratory failure (95\% [38/40] vs. $22 \%$ [9/41], $P<0.001)$ and intensive care unit admission (77.5\% [31/40] vs. $4.8 \%$ [2/41]; $P<0.001)$. The CAR-T group had higher rates of hypoalbuminemia (95.1\% [39/41] vs. 80\% [32/40], $P=0.048)$ and blood transfusion (78.0\% [32/41] vs. $32.5 \%$ [13/40], $P<0.001)$.

Table 3 and Supplement Table 2 show the results of the univariate and multivariate analyses for each complication. COVID-19 and max lg IL-10 were two independent prognostic factors associated with respiratory failure. Maximum CRP and grade 4 CRS were two independent prognostic factors associated with renal dysfunction. Maximum $\mathrm{lg}$ ferritin was an independent prognostic factor associated with hepatic dysfunction. Maximum lg D-dimer was an independent prognostic factor associated with secondary infection. Maximum lg IL-6 was an independent prognostic 
Fig. 4 Correlation between viral load/tumor burden and cytokine levels with grade 3-4 CRS in COVID-19 group $(n=40)$ and CAR-T group $(n=41)$. Pearson correlation and linear regression were used for statistical analysis. $L D H$ lactate dehydrogenase.
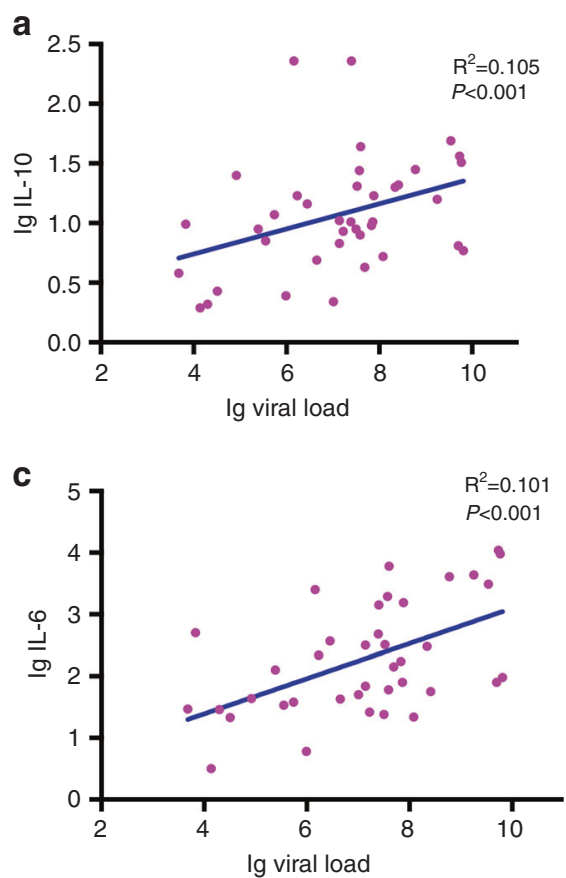
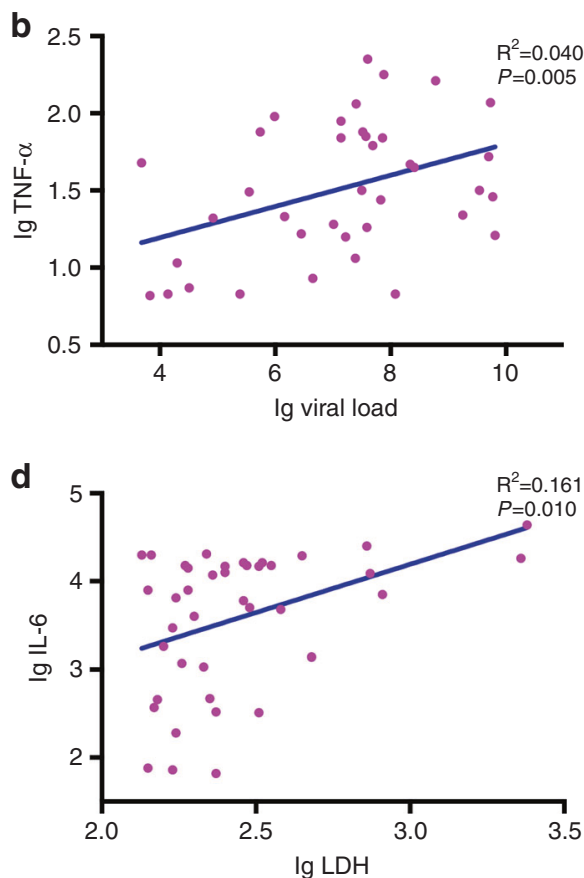

Table 4 Complications in patients with severe CRS.

\begin{tabular}{lccr}
\hline & $\begin{array}{l}\text { COVID-19 } \\
(N=40)\end{array}$ & $\begin{array}{l}\text { CAR-T } \\
(N=41)\end{array}$ & $P$ value \\
\hline Respiratory failure, $n(\%)$ & $38(95.0 \%)$ & $9(22.0 \%)$ & $<0.001$ \\
Acute cardiac injury, $n(\%)$ & $10(25.0 \%)$ & $7(17.1 \%)$ & 0.381 \\
Hepatic dysfunction, $n(\%)$ & $27(67.5 \%)$ & $34(82.9 \%)$ & 0.107 \\
Acute kidney injury, $n(\%)$ & $10(25.0 \%)$ & $13(31.7 \%)$ & 0.503 \\
Secondary infection, $n(\%)$ & $27(67.5 \%)$ & $21(51.2 \%)$ & 0.136 \\
Hypoalbuminemia, $n(\%)$ & $32(80.0 \%)$ & $39(95.1 \%)$ & 0.048 \\
Transfer to ICU, $n(\%)$ & $31(77.5 \%)$ & $2(4.8 \%)$ & $<0.001$ \\
Blood transfusion, $n(\%)$ & $13(32.5 \%)$ & $32(78.0 \%)$ & $<0.001$ \\
Gastrointestinal and & $3(7.5 \%)$ & $3(7.3 \%)$ & 1.000 \\
craniocerebral hemorrhage, $n(\%)$ & & & \\
\hline
\end{tabular}

Data were described as $n(\%) . P$ value were tested by Chi-square test. ICU intensive care unit.

factor associated with blood transfusion. None of the complications were independently related to the duration of sCRS or the procalcitonin concentration.

\section{Treatment and prognosis}

Table 5 shows the treatments for sCRS in the two groups. Patients in the COVID-19 group were more likely than patients in the CAR-T group to receive high-flow oxygen, invasive mechanical ventilation, or emergency extracorporeal membrane oxygenation $(100 \%$ [40/40] vs. $34.1 \%$ [14/41], $P<0.001)$. Furthermore, treatments in the COVID19 group tended to involve corticosteroids and/or artificial-
Table 5 Treatments in patients with severe CRS.

\begin{tabular}{|c|c|c|c|}
\hline & $\begin{array}{l}\text { COVID-19 } \\
(N=40)\end{array}$ & $\begin{array}{l}\text { CAR-T } \\
(N=41)\end{array}$ & $P$ value \\
\hline Oxygen support, $\mathrm{n}(\%)$ & & & $<0.001$ \\
\hline No need for oxygen therapy & $0(0)$ & $12(29.3 \%)$ & \\
\hline Nasal cannula & $0(0)$ & $15(36.6 \%)$ & \\
\hline $\begin{array}{l}\text { Non-invasive ventilation or } \\
\text { high-flow nasal cannula }\end{array}$ & $25(62.5 \%)$ & $12(29.3 \%)$ & \\
\hline $\begin{array}{l}\text { Invasive mechanical } \\
\text { ventilation }\end{array}$ & $4(10 \%)$ & $2(4.9 \%)$ & \\
\hline $\begin{array}{l}\text { Invasive mechanical } \\
\text { ventilation and ECMO }\end{array}$ & $11(27.5 \%)$ & $0(0)$ & \\
\hline sCRS treatment, $\mathrm{n}(\%)$ & & & $<0.001$ \\
\hline Corticosteroids only & $19(47.5 \%)$ & $5(12.2 \%)$ & \\
\hline Tocilizumab only & $0(0)$ & $20(48.8 \%)$ & \\
\hline $\begin{array}{l}\text { Corticosteroids and } \\
\text { tocilizumab }\end{array}$ & $0(0)$ & $7(17.1 \%)$ & \\
\hline $\begin{array}{l}\text { Artificial-liver blood- } \\
\text { purification only }\end{array}$ & $1(2.5 \%)$ & $0(0)$ & \\
\hline $\begin{array}{l}\text { Corticosteroids and artificial- } \\
\text { liver blood-purification }\end{array}$ & $13(32.5 \%)$ & $0(0)$ & \\
\hline
\end{tabular}

Data were described as $n(\%) . P$ value were tested by Chi-Square test and Fisher's exact test. ECMO extracorporeal membrane oxygenation.

liver blood-purification therapy, whereas pharmacological treatment in the CAR-T group tended to involve tocilizumab with or without corticosteroids. Timely treatments resulted in remission of sCRS in all patients, 40 patients in COVID-19 group were proved SARS-COV-2 negative in respiratory specimens by viral nucleic acid detection before discharge. As for CAR-T group, 9.8\% (4/41) patients failed 
to achieve complete remission after CAR-T-cell therapy. The median follow-up days were 128 days (range: 91-139 days) and 287 days (range: 12-1344 days) in COVID-19 group and CAR-T group, no deaths occurred in COVID-19 group during follow-ups. However, the relapse rate and mortality of CAR-T group was $29.3 \%$ (12/41) and $34.1 \%$ (14/41), among which four patients died because of serious complications at a median interval of 20.5 days after the CAR-T infusion (range: 13-74 days). Patient 1 with relapsed/refractory (RR) MM died because of severe pneumonia secondary to adenovirus infection at 25 days after CAR-T infusion. Patient 2 with RR MM died because of intracranial hemorrhage at 13 days after CAR-T infusion. Patient 3 with RR MM and died because of acute liver failure after hepatitis $B$ virus reactivation at 74 days after CAR-T infusion. Patient 4 with RR ALL died because of pseudomonas aeruginosa sepsis and septic shock at 16 days after CAR-T infusion.

\section{Discussion}

Several studies have evaluated the pathophysiology, clinical characteristics, and treatment strategies for CRS induced by COVID-19 or CAR-T therapy. However, there is limited information regarding the similarities and differences in these two groups. To the best of our knowledge, ours is the first study to compare the characteristics of sCRS induced by severe COVID-19 and CAR-T therapy, which may help guide the understanding, diagnosis, and treatment of COVID-19-related CRS. Our results revealed that the CAR$\mathrm{T}$ group had a more dramatic increase of serum cytokine levels, and sCRS was associated with viral load in COVID19 group and with $\mathrm{LDH}$ concentration in CAR-T group. Furthermore, COVID-19 group had a markedly higher incidence of respiratory symptoms, whereas hypotension was more common in the CAR-T group. This difference is likely related to the fact that SARS-COV-2 mainly targets the respiratory tract, whereas CAR-T cells attack hematological tumor cells disseminated throughout various organs, which causes a more intensive generalized response. The incidence of grade $4 \mathrm{CRS}$ was lower in CAR-T group, which may be attributable to the specific treatment (including tocilizumab and corticosteroids) in CAR-T group. Nevertheless, there is current no standardized treatment for COVID-19-related sCRS.

Both groups exhibited variable changes in serum concentrations of IL-2, IL- 6 , IL-10, IFN- $\gamma$, TNF- $\alpha$, ferritin, and D-dimer. CAR-T group had higher values for the laboratory parameters, with the notable exception of TNF- $\alpha$, which is likely explained by SARS-COV-2 targeting the pulmonary alveolar epithelial and endothelial cells and leading to elevated TNF- $\alpha$ levels in the lung tissues [23, 24]. Further studies are needed to test this hypothesis by comparing cytokine concentrations in bronchial alveolar lavage fluid and serum specimens. We presumed that CRS induced by COVID-19 or CAR-T therapy involves similar processes with different intensities, which suggested that the management of CAR-T-related sCRS may help guide the management of COVID-19-related sCRS.

Nevertheless, our study found that sCRS induced by COVID-19 and CAR-T therapy exhibited distinct clinical phenomenon, thus, etiologies of sCRS between the two groups may be different. The pathophysiology of CAR-Trelated CRS involves the anti-tumor activity of CAR-T cells, whereas COVID-19-related CRS involves SARSCOV-2 binding to and impairing alveolar epithelial cells, which induces cytokines secretion from the large amounts of immune cells activated by the innate and adaptive immune systems $[25,26]$. Our findings indicate that sCRS was related to a high viral load in COVID-19 cases and to a high tumor burden in CAR-T cases. In this context, CAR-T cells may have greater specificity and efficacy for targeting tumor cells, which might contribute to the more intense responses and rapid clinical outcomes of CAR-T-related CRS. However, compared to CAR-T cells, immune cells are less specificity in targeting SARS-COV-2. Recent research has indicated that COVID-19 patients have dramatically decreased counts of CD4+ and CD8 + T cells, especially in patients who are treated in the intensive care unit, and T-cell counts were negatively associated with serum concentrations of IL-6, IL-10, and TNF- $\alpha$ [27, 28]. Our laboratory findings also revealed general reductions in the counts for total $\mathrm{T}$ cells, CD3 $+\mathrm{CD} 4+\mathrm{T}$ cells, and CD3 $+\mathrm{CD} 8+$ $\mathrm{T}$ cells in COVID-19 patients. Thus, CAR-T patients may experience a shorter duration of CRS than COVID-19 patients, the lack of a significant difference in our findings might be related to the small sample sizes and different treatment strategies, especially the lower rate of corticosteroids application.

The treatments for CAR-T-related sCRS were based on its severity, and mainly involved tocilizumab and corticosteroids. Tocilizumab targets IL-6 receptor and was approved by the FDA for treating grade $\geq 3$ CRS based on a reliable treatment response [29, 30]. Corticosteroids are recommended as a second-line treatment because of their potential effects on CAR-T-cell proliferation and efficacy, and should be used for patients with disease that is refractory to tocilizumab treatment $[3,31]$. Centers that administer CAR-T therapy have established systematic strategies for reducing the incidence and mortality of CAR-T-related CRS. However, tocilizumab has not been officially approved for treating sCRS related to COVID-19. Several clinical trials (NCT04331794, NCT04377659 et al) have evaluated tocilizumab in COVID-19 patients with severe or critical disease with extensive lung lesions, the results revealed a 
decreased fraction of inspired oxygen, reduced lung lesion opacity, and recovery of lymphoma proportion and CRP concentrations after tocilizumab therapy [32]. Thus, taking into account the lower serum IL-6 concentration in COVID19 group, we suspect that careful treatment with a conservative dose and treatment period may be useful.

Our study displayed a significantly higher concentration of TNF- $\alpha$ in the COVID-19 group, which consistents with the results from previous studies. Jamilloux Y. et al. [33] founded that TNF- $\alpha$ released when viral RNA is recognized by toll-like receptors, NOD-like receptors, and RIG-I like receptors. Furthermore, in SARS-COV-2-infected mice, increased TNF- $\alpha$ was a principal cause of acute lung injury, impaired T-cell function and T-cell apoptosis [31, 34]. Thus, we speculate that targeting TNF- $\alpha$ may be a novel strategy for managing sCRS that is caused by SARS-COV2 , although this theory requires further investigation.

Corticosteroid is a double-edged sword in managing immunomodulation and the inflammatory response. Although corticosteroids can lead to an increased plasma viral load in patients and cause aggravation of mild disease, previously reported findings and our results suggest that it remains useful for managing severe cases that are associated with over-secretion of cytokines [35]. The pharmacological mechanism involves corticosteroids decreasing the secretion of inflammatory factors, as well as leukocyte infiltration and phagocytosis at the beginning of inflammation. In addition, corticosteroids interfere with the interactions between proinflammatory factors [35-37]. Therefore, short-term administration of corticosteroids (e.g., 3-5 days) at early stage of cytokine storm may help relieve syndromes and delay disease progression $[38,39]$. In the present study, corticosteroids were provided to most of the patients with COVID-19-related sCRS (32/40 cases, 80\%), which resulted in a lower temperature, shorter fever duration, and lower serum cytokine concentrations. These results may indicate that corticosteroids are a valuable treatment for critically ill patients with sCRS.

Patients with RR hematologic malignancies often suffer from high tumor burden and rapidly progressed disease, and to some extent, timely manufacturing and infusion of CAR$\mathrm{T}$ cells is a prospective strategy for those high-risk patients with aggressive disease. Regrettably, COVID-19 pandemic may set challenges and obstruct the smooth performing of CAR-T-cell therapy, and patients with RR hematologic malignancies and COVID-19 may encounter increased risks of CAR-T-cell toxicity. As described above, sCRS induced by CAR-T-cell therapy and COVID-19 presented similar conditions in both clinical and laboratory manifestations, they both experienced fever, respiratory symptoms, increasing of cytokines, and inflammation indicators. Furthermore, acquired humoral immunity deficiencies (like Bcell aplasia) and myelosuppression after CAR-T-cell infusion possibly increased susceptibility to COVID-19 in those patients, likewise, virus infection is a risk factor associated with cytopenia. These comparable performances and interactions between the two courses pose particular challenges in identification and diagnosing comorbid COVID-19 and hematological malignancies [40]. Previous reports showed the lack of proved treatments available for treating immunocompromised patients with COVID-19 [41]. Wei J. et al. reported a MM patient infected with SARS-COV-2 3 month after fully humanized, anti-BCMA CAR-T-cell therapy, he underwent progression of COVID19 with the elevated levels of cytokine profiles and B-cell aplasia. Unfortunately, after polypharmacy strategy of corticosteroids, cefoperazone/sulbactam, teicoplanin and voriconazole, these patients still maintain a high viral load and died of respiratory failure [40]. Therefore, some recommendations may contribute to reasonable decision-making of CAR-T-cell therapy under the circumstance of COVID19 pandemic. First, the potential risks and benefits of promoting CAR-T-cell therapy should be taken into careful consideration, and it would be reasonable to select patients with lower tumor burden and simultaneously lower risk of CRS. Second, CAR-T patients complicated with fever or respiratory symptoms need timely examine for etiology (including SARS-COV-2) and lung radiologic abnormalities. Finally, the treatment of CAR-T patients complicated with COVID-19 requires specialized expertize and a multidisciplinary team, and based on Center for Disease Control and Prevention (CDC) and ASTCT guidelines [42]. In addition, tocilizumab, TNF- $\alpha$ inhibitors, or artificial liver treatment is proposed for severe COVID-19 patients with high level of cytokines.

The present study has several limitations that must be acknowledged. First, only a small number of sCRS cases were available for the COVID-19 and CAR-T groups. Although the small sample sizes may influence the statistical power of our findings, we collected data from each stage during disease progression to improve the reliability of our conclusions. Second, the small number of cases made it impossible to compare sCRS in the COVID-19 group with CAR-T-related CRS according to the different hematological diseases. Third, additional basic research is needed to explain the underlying mechanisms for the relationships we observed in the patients' clinical characteristics and laboratory findings.

\section{Conclusion}

The global COVID-19 pandemic has created an urgent need for effective and precise treatments. In this setting, sCRS is associated with life-threatening complications, including acute respiratory distress syndrome and multiple organ 
dysfunction, which suggests that early recognition and control of sCRS will be crucial for improving outcomes among patients with severe COVID-19. Our results suggest that inflammatory responses are similar but of different intensities in sCRS that is caused by severe COVID-19 and CAR-T therapy. Both groups exhibited variable increases in different cytokines (including IL-6, IL-10, IL-2, TNF- $\alpha$, and IFN- $\gamma$ ), only TNF- $\alpha$ concentration was significantly higher in the COVID-19 group. Thus, the diagnosis and treatment of CAR-T-related sCRS may be useful for guiding the diagnosis and treatment of COVID-19-related sCRS. Moreover, further studies are needed to evaluate the potential feasibility of TNF- $\alpha$ inhibitors in this setting.

Acknowledgements The authors would like to thank the patients and families for participating in this study, the help of medical and nurse team on patients' care and department of laboratory for their help in analyzing the viral load. This work was supported by grants from the National Natural Science Foundation of China (81230014, 81470341, $81520108002)$, the Key Project of Science and Technology Department of Zhejiang Province (2018C03016-2), and the Key Research and Development Program of Zhejiang Province (2019C03016).

Author contributions H.H., Y.H., and G.W. designed and oversaw the study. R.H. and H.Z. were responsible for writing the manuscript. R. H., H.Z, and Y.W. collected and analyzed all data. Y.C. contribute to the laboratory testing, H.C. participated in sample collection and/or treatment of COVID-19 patients. All authors approved the final version of the manuscript.

\section{Compliance with ethical standards}

Conflict of interest The authors declare that they have no conflict of interest.

Publisher's note Springer Nature remains neutral with regard to jurisdictional claims in published maps and institutional affiliations.

\section{References}

1. Moore JB, June $\mathrm{CH}$. Cytokine release syndrome in severe COVID-19. Science 2020;368:473-4.

2. Oldstone MB, Rosen H. Cytokine storm plays a direct role in the morbidity and mortality from influenza virus infection and is chemically treatable with a single sphingosine-1-phosphate agonist molecule. Curr Top Microbiol Immunol. 2014;378:129-47.

3. Lee DW, Gardner R, Porter DL, Louis CU, Ahmed N, Jensen M, et al. Current concepts in the diagnosis and management of cytokine release syndrome. Blood 2014;124:188-95.

4. Pedersen SF, Ho YC. SARS-CoV-2: a storm is raging. J Clin Invest. 2020;130:2202-5.

5. Shimabukuro-Vornhagen A, Gödel P, Subklewe M, Stemmler HJ, Schlößer HA, Schlaak M, et al. Cytokine release syndrome. J Immunother Cancer. 2018;6:56.

6. Yáñez L, Sánchez-Escamilla M, Perales MA. CAR T cell toxicity: current management and future directions. Hemasphere. 2019;3: e186.

7. Maude SL, Barrett D, Teachey DT, Grupp SA. Managing cytokine release syndrome associated with novel $\mathrm{T}$ cell-engaging therapies. Cancer J. 2014;20:119-22.
8. Majzner RG, Mackall CL. Clinical lessons learned from the first leg of the CAR T cell journey. Nat Med. 2019;25:1341-55.

9. Schuster SJ, Bishop MR, Tam CS, Waller EK, Borchmann P, McGuirk JP, et al. Tisagenlecleucel in adult relapsed or refractory diffuse large B-cell lymphoma. N Engl J Med. 2019;380:45-56.

10. Park JH, Rivière I, Gonen M, Wang X, Sénéchal B, Curran KJ, et al. Long-term follow-up of CD19 CAR therapy in acute; ymphoblastic leukemia. N Engl J Med. 2018;378:449-59.

11. Ali SA, Shi V, Maric I, Wang M, Stroncek DF, Rose JJ, et al. $\mathrm{T}$ cells expressing an anti-B-cell maturation antigen chimeric antigen receptor cause remissions of multiple myeloma. Blood. 2016;128:1688-700.

12. Mosser DM, Edwards JP. Exploring the full spectrum of macrophage activation. Nat Rev Immunol. 2008;8:958-69.

13. Hunter BD, Jacobson CA. CAR T-cell associated neurotoxicity: mechanisms, clinicopathologic correlates, and future directions. J Natl Cancer Inst. 2019. https://doi.org/10.1093/jnci/djz017.

14. Xiong Y, Liu Y, Cao L, Wang D, Guo M, Jiang A, et al. Transcriptomic characteristics of bronchoalveolar lavage fluid and peripheral blood mononuclear cells in COVID-19 patients. Emerg Microbes Infect. 2020;9:761-70.

15. Agarwal S, June CH. Harnessing CAR T-cell insights to develop treatments for hyperinflammatory responses in patients with COVID-19. Cancer Discov. 2020;10:775-8.

16. de Wit E, van Doremalen N, Falzarano D, Munster VJ. SARS and MERS: recent insights into emerging coronaviruses. Nat Rev Microbiol. 2016;14:523-34.

17. Pascarella G, Strumia A, Piliego C, Bruno F, Del Buono R, Costa F, et al. COVID-19 diagnosis and management: a comprehensive review. J Intern Med. 2020. https://doi.org/10.1111/joim.13091.

18. England JT, Abdulla A, Biggs CM, Lee AYY, Hay KA, Hoiland RL, et al. Weathering the COVID-19 storm: lessons from hematologic cytokine syndromes. Blood Rev. 2020. https://doi.org/10. 1016/j.blre.2020.100707.

19. Huang C, Wang Y, Li X, Ren L, Zhao J, Hu Y, et al. Clinical features of patients infected with 2019 novel coronavirus in Wuhan, China. Lancet 2020;395:497-506.

20. Guan WJ, Ni ZY, Hu Y, Liang WH, Ou CQ, He JX, et al. Clinical characteristics of coronavirus disease 2019 in China. N Engl J Med. 2020;382:1708-20.

21. Diagnosis and Treatment Plan of COVID-19 (5th Trial Edtion, Revised). Chin J Integr Med. 2020;40:136-8.

22. Common Terminology Criteria for Adverse Events (CTCAE) Version 5.0; 2017.11.27:51.

23. Haga S, Yamamoto N, Nakai-Murakami C, Osawa Y, Tokunaga $\mathrm{K}$, Sata T, et al. Modulation of TNF-alpha-converting enzyme by the spike protein of SARS-CoV and ACE2 induces TNF-alpha production and facilitates viral entry. Proc Natl Acad Sci USA. 2008;105:7809-14.

24. Jamilloux Y, Henry T, Belot A, Viel S, Fauter M, Jammal T. El, et al. Should we stimulate or suppress immune responses in COVID-19? Cytokine and anti-cytokine interventions. Autoimmun Rev. 2020;19:1-13.

25. Leiva-Juárez MM, Kolls JK, Evans SE. Lung epithelial cells: therapeutically inducible effectors of antimicrobial defense. Mucosal Immunol. 2018;11:21-34.

26. Kirkpatrick CT, Wang Y, Leiva Juarez MM, Shivshankar P, Pantaleón García J, Plumer AK, et al. Inducible lung epithelial resistance requires multisource reactive oxygen species generation to protect against viral infections. mBio 2018;9:1-18.

27. Diao B, Wang C, Tan Y, Chen X, Liu Y, Ning L, et al. Reduction and functional exhaustion of $\mathrm{T}$ cells in patients with coronavirus disease 2019 (COVID-19). Front Immunol. 2020;11:827.

28. Xu B, Fan CY, Wang AL, Zou YL, Yu YH, He C, et al. Suppressed $\mathrm{T}$ cell-mediated immunity in patients with COVID-19: a 
clinical retrospective study in Wuhan, China. J Infect. 2020. https://doi.org/10.1016/j.jinf.2020.04.012.

29. Le RQ, Li L, Yuan W, Shord SS, Nie L, Habtemariam BA, et al. FDA approval summary: tocilizumab for treatment of chimeric antigen receptor $t$ cell-induced severe or life-threatening cytokine release syndrome. Oncologist 2018;23:943-7.

30. Hay KA. Cytokine release syndrome and neurotoxicity after CD19 chimeric antigen receptor-modified (CAR-) T cell therapy. Br J Haematol. 2018;183:364-74.

31. Neelapu SS, Tummala S, Kebriaei P, Wierda W, Gutierrez C, Locke FL, et al. Chimeric antigen receptor T-cell therapy assessment and management of toxicities. Nat Rev Clin Oncol. 2018;15:47-62.

32. Zhang S, Li L, Shen A, Chen Y, Qi Z. Rational use of tocilizumab in the treatment of novel coronavirus pneumonia. Clin Drug Investig. 2020;40:511-8.

33. Jamilloux Y, Henry T, Belot A, Viel S, Fauter M, El Jammal T, et al. Should we stimulate or suppress immune responses in COVID-19? Cytokine and anti-cytokine interventions. Autoimmun Rev. 2020;19:1-13.

34. McDermott JE, Mitchell HD, Gralinski LE, Eisfeld AJ, Josset L, Bankhead A 3rd, et al. The effect of inhibition of PP1 and TNFo signaling on pathogenesis of SARS coronavirus. BMC Syst Biol. 2016;10:93.

35. Yang Z, Liu J, Zhou Y, Zhao X, Zhao Q, Liu J. The effect of corticosteroid treatment on patients with coronavirus infection: a systematic review and meta-analysis. J Infect. 2020. https://doi. org/10.1016/j.jinf.2020.03.062.

36. Busillo JM, Cidlowski JA. The five Rs of glucocorticoid action during inflammation: ready, reinforce, repress, resolve, and restore. Trends Endocrinol Metab. 2013;24:109-19.

37. Bhattacharyya S, Brown DE, Brewer JA, Vogt SK, Muglia LJ. Macrophage glucocorticoid receptors regulate Toll-like receptor 4-mediated inflammatory responses by selective inhibition of $\mathrm{p} 38$ MAP kinase. Blood. 2007;109:4313-9.

38. Qin YY, Zhou YH, Lu YQ, Sun F, Yang S, Harypursat V, et al. Effectiveness of glucocorticoid therapy in patients with severe coronavirus disease 2019: protocol of a randomized controlled trial. Chin Med J. 2020;133:1080-6.

39. Boni C, Barili V, Acerbi G, Rossi M, Vecchi A, Laccabue D, et al. HBV immune-therapy: from molecular mechanisms to clinical applications. Int J Mol Sci. 2019;20:2754.

40. Y Hu, E Tan Su Yin, Y Yang, H Wu, G Wei, J Su, et al. CAR Tcell treatment during the COVID-19 pandemic: management strategies and challenges. Curr Res Transl Med. 2020, 68:111-8.

41. Wei J, Zhao J, Han M, Meng F, Zhou J. SARS-CoV-2 infection in immunocompromised patients: humoral versus cell-mediated immunity. J Immunother Cancer. 2020;8:1-8.

42. ASTCT INTERIM PATIENT GUIDELINES APRIL 20, 2020. Available from: https://www.astct.org/viewdocument/astctinterim-patient-guidelines-ap?CommunityKey $=\mathrm{d} 3949 \mathrm{~d} 84$ 3440-45f4-814290ea05adb0e5\&tab=librarydocuments. 